\title{
Asset integrity case development for normally unattended offshore installations: Bayesian network modelling
}

\author{
S. Loughney, J. Wang \\ Liverpool John Moores University, UK \\ D. Lau, D. Minty \\ RMRI Consulting Plc., UK
}

\begin{abstract}
This research proposes the initial stages of the application of Bayesian Networks in conducting quantitative risk assessment of the integrity of an offshore system. The main focus is the construction of a Bayesian network model that demonstrates the interactions of multiple offshore safety critical elements to analyse asset integrity. A NUI (Normally Unattended Installation) - Integrity Case will enable the user to determine the impact of deficiencies in asset integrity and demonstrate that integrity is being managed to ensure safe operations in situations whereby physical human to machine interaction is not occurring. The Integrity Case can be said to be dynamic as it shall be continually updated for an installation as the Quantitative Risk Analysis (QRA) data is recorded. This allows for the integrity of the various systems and components of an offshore installation to be continually monitored. The Bayesian network allows cause-effect relationships to be modelled through clear graphical representation. The model accommodates for continual updating of failure data.
\end{abstract}

\section{INTRODUCTION}

This research focuses on the development of an Initial Bayesian Network (BN) model for modelling system and component failures on a large offshore installation. The intention of the presented research is to model a sequence of events following a specific component failure, under certain conditions and assumptions. This sequence of events is then applied to a $\mathrm{BN}$ model using a proposed methodology. This should provide a base with which to expand the BN model to facilitate the requirement of having a dynamic risk assessment model within an NUI (Normally Unattended Installation) - Integrity Case.

An Asset Integrity Case will enable the user to determine the impact of deficiencies in asset integrity on the potential loss of life and demonstrate that integrity is being managed to ensure safe operations. The Integrity Case is an extended Safety Case. Where safety cases demonstrate that safety procedures are in place, the Integrity Case shall ensure that the safety procedures are properly implemented. The Integrity Case can be applicable to operations for any large scale asset, and in the case of this research the large asset for which the Integrity Case shall be developed is an offshore installation (RMRI Plc., 2011). By expanding on this Integrity Case proposal, it is intended that an Integrity Case be developed for a Normally Unattended Installation (NUI) in conjunction with a dynamic risk assessment model to maintain a live representation of an offshore installations integrity. Furthermore, it is proposed that the NUI-Integrity Case be initially developed utilising a manned installation, but modelling failure and risks without human presence on board. This is due to a much larger range of failure data being available regarding manned installations as opposed to unmanned installations. Similarly, should a risk assessment model be feasible for various hazardous zones of an installation, and the dynamic model proves to be effective in the detection of failures and mapping of consequences, it may be possible to reduce the number of personnel on board manned offshore installations, to reduce the risk of injury and fatality.

The paper is structured as follows. Section 2 presents a brief background into the origins of the research. A proposed methodology of constructing a BN model is shown in section 3. Section 4 outlines and analyses a case study to demonstrate the proposed methodology. Section 5 summarizes the work.

\section{BACKGROUND}

\subsection{Offshore Safety Assessment}

Following the public inquiry into the Piper Alpha disaster, the responsibilities for offshore safety regulations were transferred from the Department of Energy to the Health and Safety Commission (HSC) through the Health and Safety Executive (HSE) as the singular regulatory body for safety in the offshore industry (Wang, 2002) (Department of Energy, 
1990). In response to this the HSE launched a review of all safety legislation and subsequently implemented changes. The propositions sought to replace the legislations that were seen as prescriptive to a more "goal setting" approach. Several regulations were produced, with the mainstay being the Health and Safety at Work Act (HSE, Health and Safety Executive, 1992). Under this a draft of the offshore installations safety case regulations was produced. The regulations required operational safety cases to be prepared for all offshore installations, both fixed and mobile. Within this all new fixed installations require a design safety case and for mobile installations, the duty holder is the owner (Wang, 2002).

After many years of employing the safety case approach in the UK offshore industry, the regulations were expanded in 1996 to include verification of Safety Critical Elements (SCEs). Also the offshore installations and wells regulations were introduced to deal with various stages of the life cycle of the installation. SCEs are parts of an installation and its plant, including computer programs or any part whose failure could cause or contribute substantially to or whose purpose of which is to prevent or limit the effect of a major accident (Wang, 2002) (HSE, Health and Safety Executive, 1996).

Recently, however, it is felt that an expansion on Safety Cases is necessary, especially in the offshore and marine industry, as they are static documents that are produced at the inception of offshore installations and contains a structured argument demonstrating that the evidence contained therein is sufficient to show that the system is safe (Auld, 2013). However, this is the full extent of the Safety Case, it involves very little updating unless an operational or facility change is made. It can be difficult to navigate through a safety case; they can be difficult for project teams and regulators to understand, as well as often being monolithic (Risktec, 2013). This is where the e-Safety Case comes into play. e-Safety Cases are html webbased electronic Safety Cases. They are much easier to navigate and have clear concise information about the safety of the facility they are provided for. However, the QRA data (Quantified Risk Assessment) is only updated with the release of updated regulations (Cockram \& Lockwood, 2003). Over the past 10 years it has been stated that a dynamic risk assessment model is required within the offshore and process industries. Khakzad, et al., (2013) proposed to apply BN to Bow-Tie (BT) analysis. They postulated that the addition of $\mathrm{BN}$ to BT would help to overcome the static limitations of BT and show that the combination could be a substantial dynamic risk assessment tool. Similarly, in the oil, gas \& process industry Yang \& Mannan, (2010) proposed a methodology of Dynamic operational Risk Assessment (DORA). This starts from a conceptual framework design to mathematical modelling and to decision making based on costbenefit analysis. Furthermore, Eleye-Datubo, et al. (2006) proposed an offshore decision-support solution, through $\mathrm{BN}$ techniques, to demonstrate that it is necessary to model the assessment domain such that the probabilistic measure of each event becomes more reliable in light of new evidence being received. This method is prefered, as opposed to obtaining data incrementally, causing uncertainty from imperfect understanding and incomplete knowledge of the domain being analysed. Finally, RMRI Plc. (2011) proposed the idea of a dynamic decision making tool in an Asset Integrity Case.

The Integrity Case, an idea proposed by RMRI Plc. (Risk Management Research Institute), can be said to be dynamic as it shall be continually updated with the QRA data for an installation as the QRA data is recorded. This allows for the integrity of the various systems and components of a large asset, such as an offshore installation, to be continually monitored. This continual updating of the assets QRA data allows for the users to have a clearer understanding of the current status of an asset. It also allows the user to identify the impact of any deviation from specified performance standards, as well as facilitate more efficient identification of appropriate risk reduction measures, identify key trends within assets (i.e.: failures, failure modes). Reporting to regulators would improve greatly and it would provide a historical audit trail for the asset. Furthermore, the integrity of an asset is maintained so that potential loss of life is kept ALARP. This means that an asset may continue safe operations under circumstances that may have instigated precautionary shutdown, resulting in considerable cost savings for the owner and operator (RMRI Plc., 2011).

\section{METHODOLOGY}

\subsection{Modelling and Analysis Steps}

There are many step-by-step procedures in use that allow for construction of the various parts of the $\mathrm{BN}$ model. The procedures are useful as it allows for maintaining consistency throughout the process and offers an element of confidence to the model. The procedures have varying parts depending on the context of the model and how much information is already available (Neil, et al., 2000). However, there are key elements which all the procedures follow, these are:

\subsubsection{Establish the domain and project definition}

This involves putting boundaries in place for the model. In this analysis the domain is to be defined as a module on a large offshore installation. The model begins with an initial component failure and tracks 
the cause and effect relationship of this failure on various other components and systems. The model ends with outlined consequences. The objective of the model involves stating what results are expected to be achieved from the model. For the model in this research the focus is on the interaction of the components and their probability of occurrence.

\subsubsection{Identify the set of variables relative to the problem}

This involves filtering possible parameters that are relevant to the description and objective. For the model the initial variables were devised utilising a sequence of events diagram. This sequence of events diagram represents the steps of various events with their order and causality. The events in the diagram are connected with arcs and arrows. This allows for a straightforward transition to a $\mathrm{BN}$.

\subsubsection{Form Nodes and Arcs for the BN}

The events and consequences in the sequence of events are translated to corresponding parent and child nodes in the Bayesian Network. The sequence of events, however, is basic and the arcs do not directly translate to the $\mathrm{BN}$ and are determined in Step 4. The nodes can be expressed as positive or negative. The causality between the events is translated to corresponding Conditional Probability Tables (CPTs). The CPTs are constructed in Step 5. Once the relevant nodes are identified, they are input into a BN software package, HuginResearcher7.7, and connected. This entails referring to the sequence of events from the initial failure to determine the most effective way of connecting the nodes together. The network is reviewed to ensure there are no missing factors.

\subsubsection{Data acquisition and analysis}

Primarily, data is sought from various sources including: industrial \& academic publications, offshore risk assessment projects, as well as databases such as; the Offshore Reliability Database (OREDA), HSE \& the International Association of Oil and Gas Producers database (OGP). However, should data not be widley available or the CPT for a node be much to large to constuct utilising data from the outlined sources, then expert judgement is to be utilised. The expert judgement is to be obtained using the Pairwise Comparision (PC) technique and analysed with the Analytical Hierarchy Process (AHP). The data from the AHP analysis is translated to the CPTs using a Symmetric Method. The data from relevent sources is then used to create the marginal or conditional probability tables.
Analysis

This step concerns itself with the analysis of the BN model using Bayesian Inference. The probability of failure on demand of the operation is obtained by forward analysis. The posterior probabilities of the influencing factors can be calculated through backward analysis, given some evidence entered into the model. the propogation of the $\mathrm{BN}$ is conducted using Hugin Researcher 7.7. The results of the analysis provide useful information in handling the the effect of one failure on multiple components and systems. These results are demonstrated through a Sensitivity Analysis. The data for this analysis is again produced by the Hugin Researcher 7.7 software.

\subsubsection{Validation of the BN Model}

Validation is a key aspect of the methodology as it provides a reasonable amount of confidence to the results of the model. In carrying out a full validation of the model, the parameters should be closely monitored for a given period of time. For modelling a specific failure within an electrical generator, this exercise is not practical. In current work and literature, there is a three axiom based validation procedure, which is used for partial validation of the proposed BN model. The three axioms to be satisfied are as follows (Jones, et al., 2010):

\section{Axiom $i$ :}

A small increase or decrease in the prior subjective probabilities of each parent node should certainly result in the effect of a relative increase or decrease of the posterior probabilities of the child node.

\section{Axiom ii:}

Given the variation of subjective probability distributions of each parent node, its influence magnitude to the child node should be kept consistent.

\section{Axiom iii:}

The total influence magnitudes of the combination of the probability variations from " $x$ " attributes (evidence) on the values should always be greater than that from the set of " $\mathrm{x}-\mathrm{y}$ " ( $\mathrm{y} \in \mathrm{x})$ attributes. 


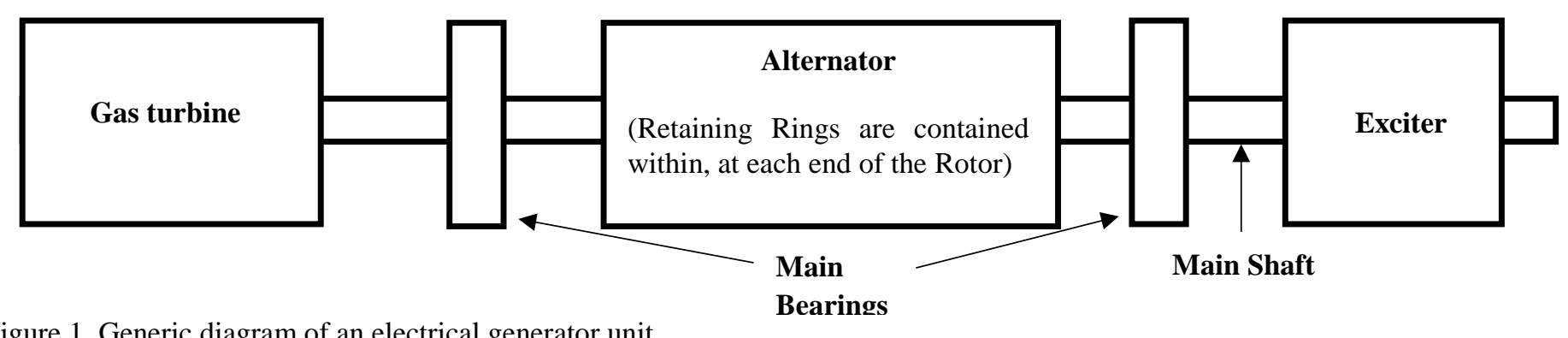

Figure 1. Generic diagram of an electrical generator unit

From this the most likely point of failure within the

\section{CASE STUDY}

\subsection{Establish domain and model definition}

In order to demonstrate the proposed methodology a case study is used to evaluate of the effects a rotor retaining ring failure has on an offshore electrical generation unit. As well as other key systems, within and adjacent to a module of a large offsore installation.

The electrical generation unit is considered to be of a generic layout for electrical generation on a large platform. The generator consists of a primary alternator, driven by a gas turbine. Located after the alternator is the exciter. The alternator rotor and shaft are forged in one piece with the exciter coupled on to one end. The opposite end of the shaft is coupled to the turbine drive shaft, which has an approximate operating speed of $3,600 \mathrm{rpm}$. The main shaft is supported by two main bearings, housed in pedastals, on stools on the baseplate. One bearing is situated between the turbine and the alternator and the other between the alternator and the exciter. A generic flowdiagram of an electrical generation unit is illustrated by Figure 1.

\subsection{Identifying the set of variables relative to the problem}

The variables are identified based upon the failure of one specific component, in this case a Rotor Retaining Ring. Should one of the retaining rings fail, the main shaft would become unbalanced causing potential fragmentation of the rings inside the alternator. Given the extreme tolerances' within the generator construction, the unbalanced shaft could also cause damage to other areas of the equipment, such as: the turbine blades and the exciter. Should the retaining ring fail within the alternator casing and fragment, debris would be created within the casing. Furthermore with the machine operating at approximately 3,600 rpm, an out of balance shaft would cause substantial vibrations, which could cause the main bearings to fail. Should the bearings fail, causing the shaft to become misaligned, it would result in increased damage to the turbine, alternator and exciter (RMRI Plc., 2009). turbine is the turbine blades shearing. Multiple blade failure could lead to the turbine casing not fully containing the turbine blade debris. This would result in turbine blades being expelled through the turbine casing as high velocity projectiles. Continually, the violent shaft vibrations and misalignment could have a severe impact on the exciter and may result in the exciter, weighing approximately one tonne, becoming detached from the main shaft. Some catastrophic failures have resulted in the exciter breaking up and some have had the exciter remain mostly intact (RMRI Plc., 2009). Should the bearings not fail, the alternator stator coils \& casing, can provide enough resistance and are substantial enough to prevent the debris from the retaining ring penetrating the alternator casing. However, it is possible for the fragments to be expelled axially towards either the turbine or the exciter or both. (U.S. Nuclear Regulatory Commission, 2008).

In the event of one or two rotor retaining ring failures, significant damage could occur within the alternator casing and fragments of the retaining ring could be expelled axially. Should the ring debris be expelled, it is assumed that it will travel in two possible direction; i) towards the turbine or ii) towards the exciter and out of the casing. Should the debris travel to the turbine there is potential for the fragments to impact the fuel gas line within the turbine. This then provides the escalation to a fire (given the location of the potential release, ignition is assumed). Should the debris travel out of the casing towards the exciter, it is considered by RMRI. Plc (2009) that while the axial velocity may be considerable, it is likely to be lower than the radial velocity that the debris would be expelled at were the casing and stator not there. Therefore, while it is possible for the ring debris to penetrate the casing, they would not have the required velocity to penetrate the module walls or deck. From this it is deemed that if retaining ring failure does not cause a bearing failure, then the consequence of the event is likely to be limited to the damages caused by the retaining ring (U.S. Nuclear Regulatory Commission, 2008).

However, should the main bearing fail, the potential consequences become much more severe. The significant damage caused by the bearing failure can potentially produce high velocity projectiles from the turbine blades being expelled and/or the exciter becoming detached (RMRI Plc., 2009). In these 
events, there is potential for the projectiles to impact the hydrocarbon containment around the module.

\subsection{Form Nodes and Arcs for the BN}

The initial model is demonstrated in Figure 2 and is designed around the variables identified section 4.2 , and is to represent the cause and effect of one initial component failure has on systems within the stated domain. The Initial BN model is not a direct representation of the sequence of events in terms of the section of the model where possible debris is expelled. Within the sequence of events if the debris is not expelled initially, it is assumed to remain in the alternator, yet if debris expelled, it is assumed to travel towards the exciter. Similarly, should the debris not be expelled to the exciter, it is assumed to be expelled towards the turbine. While this is all possible, it is more realistic to assume that if the debris is created from the retaining ring failure, it has the potential travel to the turbine and the exciter in the same instance. However, it is possible for debris to be expelled to the exciter and not to the gas turbine, whereby some debris would remain in the alternator. The way in which the $\mathrm{BN}$ model is created ensures it contains all relevant possible outcomes.

In this case the analysis is conducted within an electrical generation module of a large offshore installation. The initial model is made up of seventeen chance nodes labelled 1 to 10 and E1 to E7. The latter nodes represent the possible events that can result from the initial mechanical failure. All nodes have two states ("Yes" and "No") except for event node E6 which has four ("Small", "Medium", "Full-bore" and "None"). The BN constructed from the variables outlined is shown in Figure 2.

\subsection{Data acquisition and analysis}

It is important to note that the numerical results of the model are not significant in terms of being absolute, but rather to serve to demonstrate the practicability of the model. Once a full set of verified data is fed into the model, the confidence level associated with planning and decision making under uncertainty will improve.

To complete the CPTs within a BN, certain data and knowledge is required regarding each specific node. For some nodes data is limited or not available. For cases where there is an absence of hard data, CPTs must be completed through subjective reasoning or the application of expert judgement. This process can be demonstrated by looking at the node "Event Escalation". This node represents the chance of escalation following key component failures. The parents of this node are: "Turbine Blades Expelled", "Exciter Detaches", "Gas Import Riser Piping Impact" and "HP Flare Drum Shell Impact". In order to put together an appropriate estimate, experts must judge the situation and provide their opinions. This data acquisition can be either qualitative or quantitative in nature. However, the child node "Event Escalation" has a CPT which is too large for an expert to simply fill with their own judgements and opinions. Therefore, an effective way to gather information, to fill these large CPTs, from

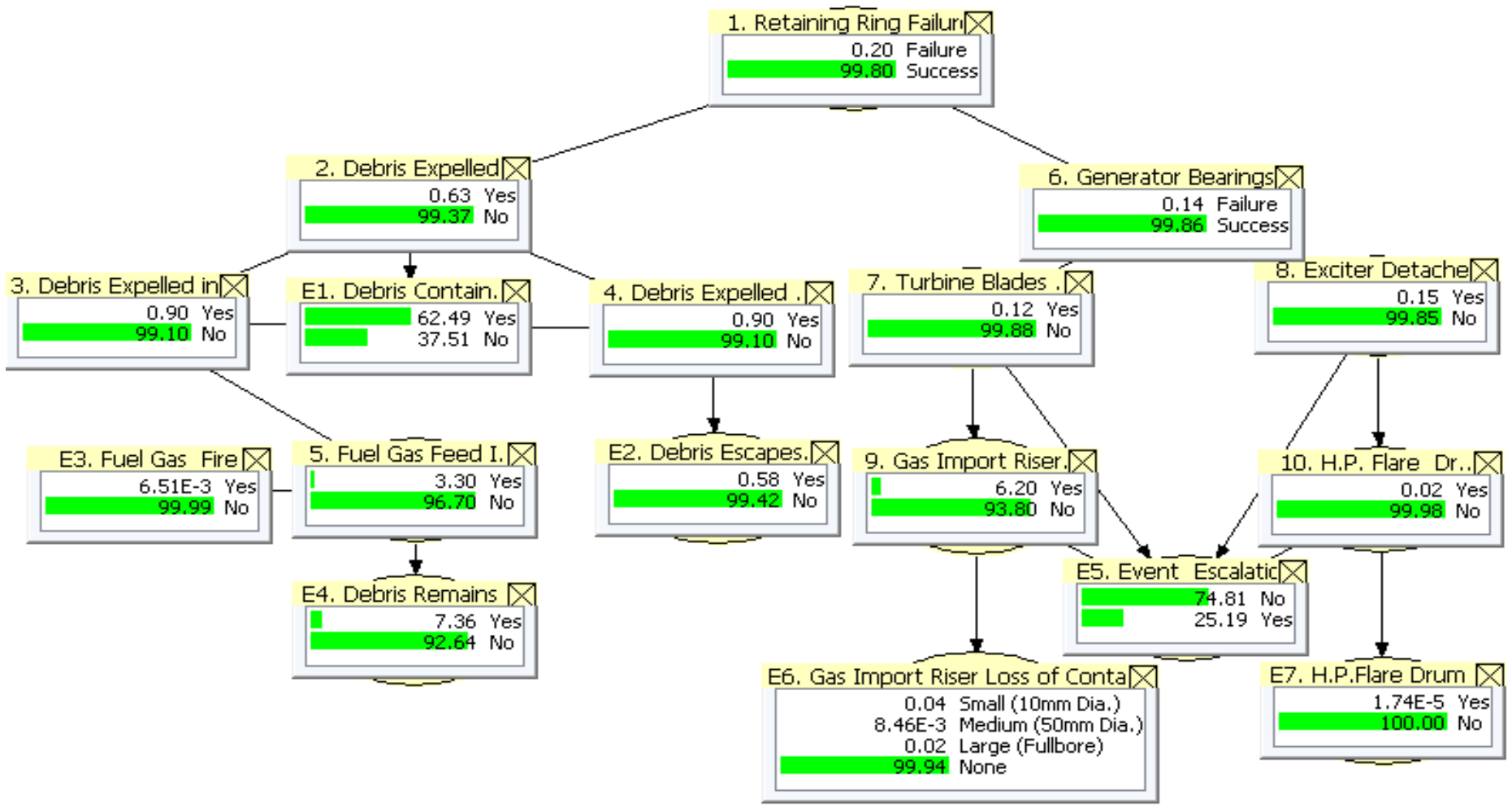

Figure 2. BN Model shown with the Marginal Probabilities for each node. 
experts is to apply the use of a Pairwise Comparison (PC) technique in questionnaires and make use of the Analytical Hierarchy Process (AHP) to analyse the results, combined with the symmetric method algorithm to fill the large CPTs (Zhang, et al., 2014).

The AHP will produce a weighting for each parent criterion in the pairwise comparison matrix. These weighting are applied to a symmetric method which is utilised to fill large CPTs. The symmetric method provides an input algorithm which consists of a set of relative weights that quantify the relative strengths of the influences of the parent-nodes on the child-node, and a set of probability distributions the number of which grows only linearly, as opposed to exponentially, with the number of associated parentnodes (Lin \& Kou, 2015) (Saaty, 1980).

The PC, AHP and symmetric methods are not to be oulined here. However, the PC and AHP methods can be found in detail in Saaty, (1980) and Koczkodaj $\&$ Szybowski., (2015). The symmetric method can be found in Das, (2008). Figure 2 shows the complete $\mathrm{BN}$ and the marginal probability distributions for each node.

\subsection{Results and Disscussions}

\subsection{1 \\ Analysis of BN model and Sensitivity}

Analysis

Quantitaive analysis is carried out on a specific section of the Initial BN model, shown in Figure 3, concerning the event "E5. Event Escalation" and its parents.

\subsubsection{Quantitative Analysis}

This analysis involved systematically inserting evidence into each of the parent nodes and finally the child node. In addition, nodes 7 and 8 have a parent node "Generator Bearings" which has no evidence inserted, and there is no evidence inserted anywhere else within the model. However, in this section of the BN model nodes 7 and 8 are parents of nodes 9 and 10 respectively, and therefore will alter the posterior probabilities of these nodes when evidence is inserted. This relationship has been left in the analysis to give an accurate representation of the posterior probabilities of the event E5, which is the focus node in this analysis. Several scenarios are considered for the $\mathrm{BN}$ analysis and validation.

The first scenario is gas turbine blades being expelled as projectiles from the generator housing. This is completed by inserting $100 \%$ to state "Yes" in node 7 . This increases the probability of the events escalating from $25.19 \%$ to $35.09 \%$. This increase would involve some concern as a potential escalation from this is the impact of the turbine blades on the Gas Import Riser. Subsequently the probability of gas import riser impact increases from $6.2 \%$ to $25 \%$.
Furthermore, the second scenario involves the expulsion of the turbine blades along with a gas riser impact (100\% "Yes" to nodes 7 and 9). This results in the probability of there being escalation increasing from $35.09 \%$ to $61.42 \%$. This is a very large increase as the impact of a gas riser is the largest threat to escalation, due to the loss of containment of the gas, this hypothesis was also confirmed by expert opinion. It can also be noted that evidence is inserted into nodes 7 and 9, there is no effect on nodes 8 and 10, which is to be expected as they should be independent from each other. Should this scenario have the potential to occur, immediate action should be taken to prevent a major accident in the form of LOC of hydrocarbons and potential explosion \& fire.

The third scenario demonstrates the potential for escalation by showing that the generator's exciter detaches, along with turbine blades expelled and gas riser impact (100\% "Yes" to nodes 7, 8 and 9). It shows that again the potential for escalation increases from $61.42 \%$ to $63.86 \%$. This scenario also increases the probability of the HP flare drum being impacted from $1.47 \%$ to $10 \%$, dues to the influence of the Exciter Detaching (represented by node 8).

Scenario five demonstrates the final influencing factor on the possibility of event escalation, whereby the HP flare drum is impacted (100\% "Yes" to nodes 7, 8, 9 and 10). This increases the potential for escalation from $63.86 \%$ to $77 \%$.
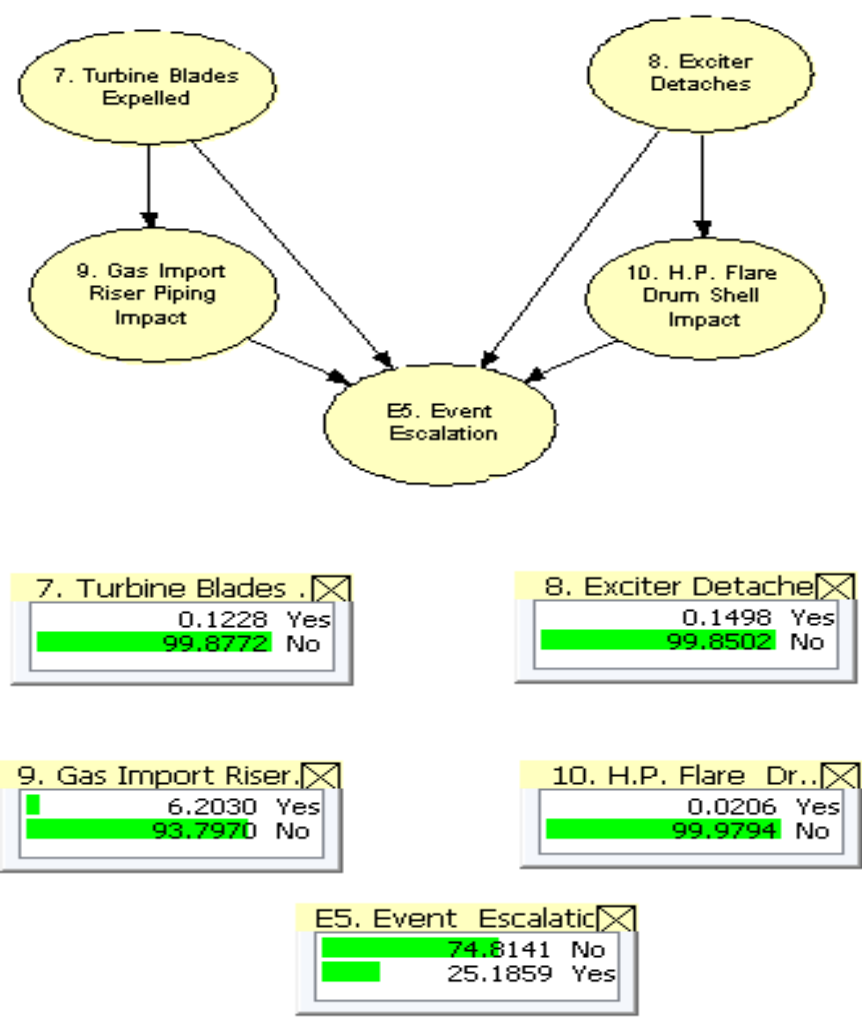

Figure 3. A) Specific section of BN to be analysed. B) Prior Probabilities for Event E5 and its parent nodes. 
The final scenario, shown in Figure 4, demonstrates the effect of there being an escalated event, for example, observing an explosion or a fire within the area of the platform containing the electrical generator, and the effect this has on the influencing parameters. This serves to obtain areas that would require closer inspection. This scenario has given insight to the possible causes of the event escalation, based upon the data presented. Here the influencing factors are: "Turbine Blades Expelled" Yes, increases from $0.12 \%$ to $0.17 \%$; "Exciter Detaches" -Yes, increases from $0.15 \%$ to $0.17 \%$; "Gas Import Riser Piping Impact" - Yes, Increases from $6.2 \%$ to $14.31 \%$; and "HP Flare Drum Shell Impact" - Yes, increasing from $0.02 \%$ to $0.03 \%$.

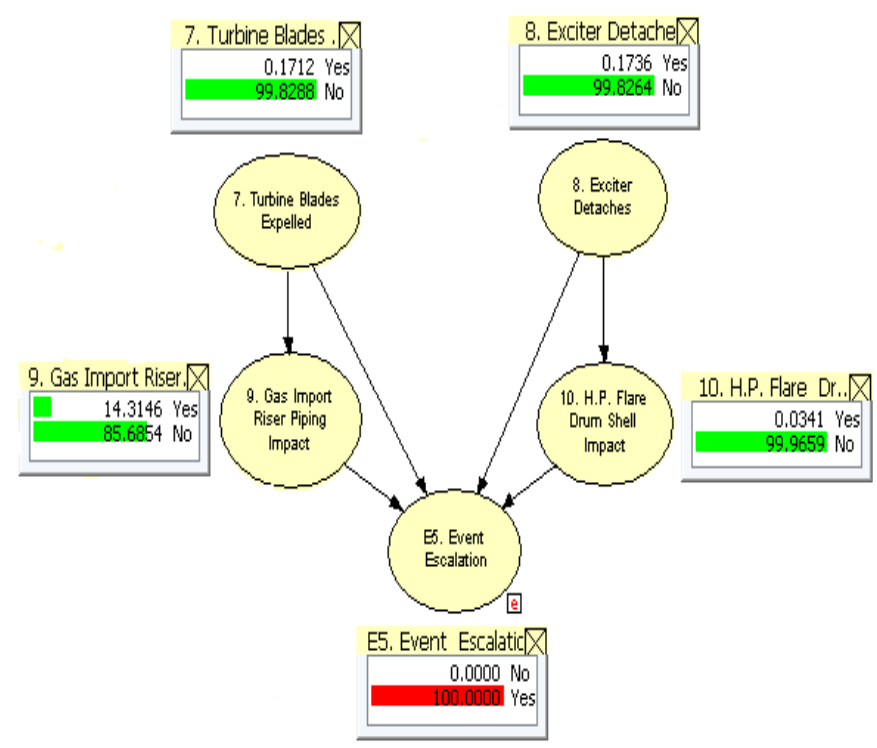

Figure 4. BN Model Illustrating when "Event Escalation" takes place.

\subsubsection{Sensitivity Analysis}

The Sensitivity Analysis conducted for the Initial BN model focuses on the event E5 and its parent nodes, shown in Figure 3, to further validate the claims in Secton 4.5.1.1. However, the analysis will be conducted using smaller increases and decreases in the probabilities of the parent nodes as opposed to inserting $100 \%$ occurrence probability into the input node CPTs.

From the graph in Figure 5 it can be seen that the most influential factor on "Event Escalation" is "Gas import Riser Impact", whilst the least influential is "Exciter Detaches". If the probability of State - 'No', "Gas Riser Impact" increases by $10 \%$, then the probability of "Event Escalation" decreases by $2.63 \%$. Whereas, if the probability of State - 'No' Detaches" increases by, "Exciter 10\%, then the probability of "Event Escalation" only decreases by $0.29 \%$. From the graph it is also apparent that the sensitivity function is a straight line which further add to the model validation. The sensitivity values computed within Hugin are shown in Table 1.

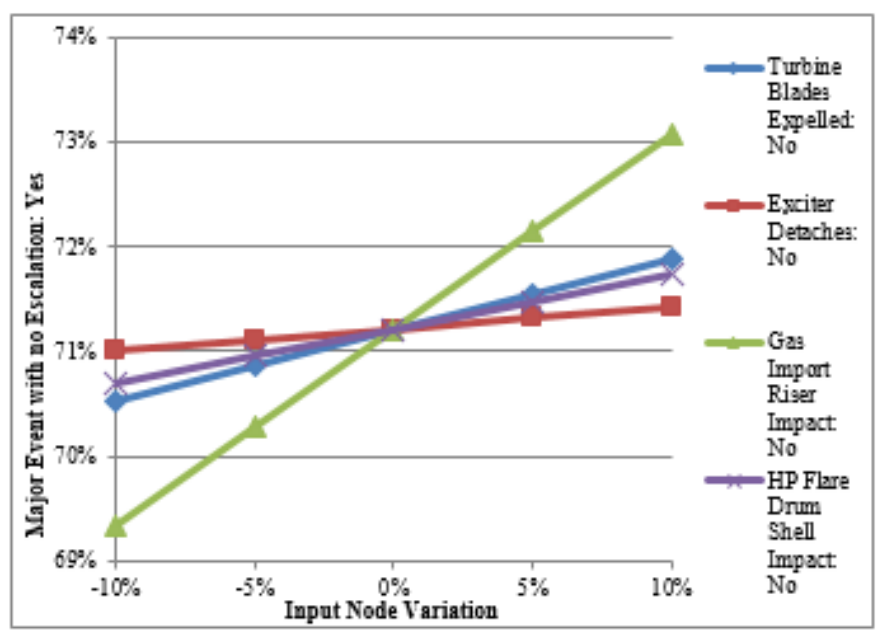

Figure 5. Sensitivity Functions for the four input nodes acting upon Event "E5. Event Escalation"

It should be noted that the sensitivity values within Table 1 are negative as in their current states of 'No', they have a negative effect on the outcome of "Event Escalation" - 'Yes'. For example; with the probability of "Turbine Blades Expelled" increasingly being 'No', it is less likely that "Event Escalation" - 'Yes' occurs.

Table 1. Sensitivity Values for the four input nodes acting upon Event "E5. Event Escalation"

\begin{tabular}{ll}
\hline Input node: "state" & Sensitivity value \\
\hline 7. Turbine blades expelled: "No" & -0.095 \\
8. Exciter detaches: "No & -0.029 \\
9. Gas import riser impact: "No" & -0.263 \\
10. HP flare drum shell impact: "No" & -0.073 \\
\hline
\end{tabular}

\subsubsection{Validation of the BN Model}

For partial validation of the model, it should satisfy the three axioms stated in Section 3.2.5. Examination of a specific part of the model (shown in Figure 3), reveals when node 7 is set to $100 \%$ 'Yes', this produces a revised increase in probability for "Event Escalation" occurring from $25.19 \%$ to $35.09 \%$. A further change including both nodes 7 and 9, set at $100 \%$ 'Yes', results in a further increase in the potential for "Event Escalation" occurring. Continually, nodes 7, 8, and 9 being set to $100 \%$ 'Yes', again results in an increase for the potential for "Event Escalation" being of the state 'Yes'.

When nodes 7, 8, 9 and 10 are set to $100 \%$ 'Yes', it produces yet another increase in the probability of "Event Escalation occurring from $63.86 \%$ to $77.00 \%$. Finally,

This exercise of increasing each of the influencing nodes satisfies the three axioms states in Section 3.2.5, thus giving partial validation to the BN Model. 


\section{CONCLUSIONS}

This research has outlined the Bayesian Network technique that has been used to model the cause and effect relationship of a specific component failure of an electrical generation system, within a module of an offshore platform. It has been stated that offshore systems can be very complex and when coupled with the volume of data required to model failures within these systems, it makes BNs a challenge to model effectively. As well as in some cases a lack of reliable data means that some risk assessment models cannot always be applied. With this in mind, the $\mathrm{BN}$ model demonstrates that BNs can provide an effective and applicable method of determining the likelihood of various events under uncertainty. The model can be used to investigate various scenarios around the systems and components outlined and to show the beginnings of establishing where attention should be focused within the objective of preventing offshore incidents, as well as having a clear representation of specifically where these accidents can originate from. The presented method of modelling offshore risk assessment is to be improved upon in future research. It has the potential to model larger areas with several systems and their components to gain a much wider understanding of how offshore systems interrelate.

There are several interesting and relevant possibilities that can be considered and explored with relative ease now that the core structure of the $\mathrm{BN}$ model has been constructed. However, before expanding the model it is vital to maintain that it must remain practical and close to reality from the perspective of gathering data and generating results. Continually too many variables which display vague information or increasingly irrelevant effects can diminish the quality of results and findings.

\section{ACKNOWLEGEMENTS}

This research is supported by Liverpool John Moores University and RMRI Plc. Thanks are also given to the EU for its financial support under a Marie Curie grant (REFERENCE - 314836; 2012 - 2016).

\section{REFERENCES}

Auld, H., 2013. A Safety Case Development Framework, Bristol: Atomic Weapons Establishment \& Defence Science and Technology Laboratory.

Cai, B. et al., 2013. Application of Bayesian Netowrks in Qunantitative Assessment of Subsea Blowout Preventer Operations. Journal of Risk Analysis, Volume 33, pp. 1293 1311.

Cockram, T. \& Lockwood, B., 2003. Electronic Safety Case: Challenges and Opportunities, s.1.: Praxis Critical Systems.

Das, B., 2008. Generating Conditional Probabilities for Bayesian Networks: Easing the Knowledge Aquisition Problem. Edinburgh, AUS: Commamd and Control Devision, DSTO.
Department of Energy, 1990. The public inquiry into the Piper Alpha Disaster, London: Department of Energy.

Eleye-Datubo, A., Wall, A., Saadjedi, A. \& Wang, J., 2006. Enabling a powerful Marine and Offshore Decision Support Solution Through Bayesian Network Technique. Risk Analysis, Volume 26, pp. 695 - 721.

HSE, Health and Safety Executive, 1992. The offshore installations (safety case) regulations, London: HSE.

HSE, Health and Safety Executive, 1996. the offshore installations and wells (design and construction, etc.) regulations, London: HSE.

HSE, Health and Safety Executive, 2006a. Guidance for Risk Assessment for Offshore installations, : HSE,.

HSE, Health and Safety Executive, 2006b. A Guide to the Offshore Installations (Safety Case) Regulations 2005, : HSE.

Jones, C., 2010. The 2010 gulf coast oil spill. 1st ed. : BookBoon.com.

Khakzad, N., Khan, F. \& Amyotte, P., 2011. Safety analysis in process facilities: comparison of fault tree and Bayesian network approaches. Reliability Engineering and System Safety, Volume 96, pp. 925-932.

Koczkodaj, W. \& Szybowski, J., 2015. Pairwise Comparison Simplified. Applied Mathematics and Computation, Volume 253, pp. 387-394.

Lin, C. \& Kou, G., 2015. Bayesian Revision of the Individual Pair-wise Comparison Matrices Under Consensus in AHPGDM. Applied Soft Computing, Volume 35, pp. 802 - 811.

Matellini, B. D., 2012. A Risk Based Fire and Rescue Management System. Liverpool: LOOM Research institute.

Risktec, 2013. Safety Case for the Offshore Wind Industry. RiskWorld, Autumn, p. 1.

RMRI Plc., 2009. Assessment of Risks Associated with the Alternator Rotor End Cap Disintegration on the Thistle Alpha Platform. Manchester: Petrofac Facilities Management Ltd..

RMRI Plc., 2011. RMRI's Asset Integrity Case, Manchester: RMRI Plc.

Saaty, T., 1980. The Analytical Hierarchy Process. NY: McGraw-Hill Book Co..

U.S. Nuclear Regulatory Commission, 2008. Potential Generator Missiles- Generator Rotor Retaining Rings. Resolution of Generic Safety Issues, August.

Wang, J., 2002. Offshore Safety Case Approach and Formal Safety Assessment of Ships. Journal of Safety Research, Volume 33, pp. 81 - 115.

Yang, X. \& Mannan, M. S., 2010. The Development and Application of Dynamic Operational Risk Assessment in Oil/Gas and Chemical Process Industry.. Journal of Reliability Engineering and System Safety, Volume 95, pp. $806-815$.

Zhang, H., Sekhari, A., Ouzrout, Y. \& Bouras, A., 2014. Deriving Consistent Pairwise Comparison Matrices in Decision Making Methodologies based on Linear Programming Method. Journal of Intelligent and Fuzzy Systems, Volume 27, pp. 1977-1989. 